\title{
CircRNA expression profiling of PBMCs from patients with hepatocellular carcinoma by RNA-sequencing
}

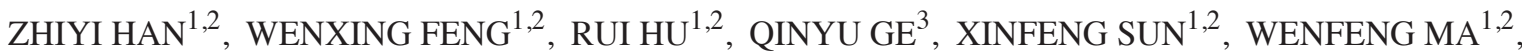

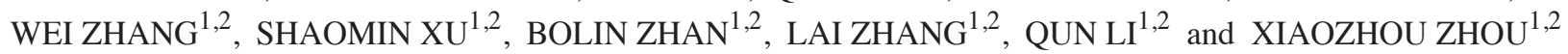 \\ ${ }^{1}$ Department of Liver Disease, Shenzhen Traditional Chinese Medicine Hospital; ${ }^{2}$ Department of Liver Disease, \\ The Fourth Clinical Medical College of Guangzhou University of Chinese Medicine, Shenzhen, \\ Guangdong 518033; ${ }^{3}$ State Key Laboratory of Bioelectronics, School of Biological Science and \\ Medical Engineering, Southeast University, Nanjing, Jiangsu 210096, P.R. China
}

Received November 4, 2020; Accepted June 22, 2021

DOI: 10.3892/etm.2021.10902

\begin{abstract}
Circular RNAs (circRNAs) are differentially expressed in various cancer types. The present study aimed to investigate the expression and clinical implication of circRNAs in hepatocellular carcinoma (HCC) and to evaluate the potential of circRNAs as diagnostic biomarkers for HCC. CircRNA expression was profiled in 19 patients with HCC and 19 normal controls using ribosomal RNA-depleted RNAs. Differentially expressed circRNAs (DE-circRNAs) between HCC and controls were identified using CIRI2 and distinct circRNA expression signatures were screened. Gene Ontology and Kyoto Encyclopedia of Genes and Genomes were used to predict the potential functions of these DE-circRNAs and the circRNA-miRNA-mRNA regulatory networks were then constructed. Several DE-circRNAs were selected and confirmed by RT-qPCR. A total of 40 DE-circRNAs (27 upregulated and 13 downregulated) were identified between patients with HCC and controls. Functional annotation indicated that these DE-circRNAs were involved in cellular components, molecular functions and cancer-associated pathways related to HCC. These included pathways in cancer, TNF signaling pathway, hepatitis B, hepatitis $\mathrm{C}$ and hepatocyte differentiation. The circRNA-miRNA-mRNA regulatory network was generated based on 11 candidate circRNAs. Receiver operating characteristic curve analysis indicated that Homo sapiens (hsa)_circ_0073239, hsa_circ_007090, hsa_circ_0008304, hsa_circ_0017586,hsa_circ_0000369 and hsa_circ_0001181 may serve as potential biomarkers for HCC. Results from Cell Counting Kit- 8 assay suggested that small interfering RNA targeting hsa_circ_0001181 reduced the proliferation
\end{abstract}

Correspondence to: Professor Xiaozhou Zhou, Department of Liver Disease, Shenzhen Traditional Chinese Medicine Hospital, 1 Fuhua Road, Futian, Shenzhen, Guangdong 518033, P.R. China E-mail: zxz1006@gzucm.edu.cn

Key words: hepatocellular carcinoma, circular RNAs, RNA-sequencing, network, reverse transcription-quantitative PCR of HepG2 cells, which implicated it as a potential therapeutic target for HCC. Therefore, in the present study, the differential expression pattern and important role of circRNAs in HCC were determined. The present results highlight the diagnostic potential of circRNAs in HCC and provide novel insight into the development of and treatment approaches for HCC.

\section{Introduction}

Hepatocellular carcinoma (HCC) is one of the most common cancer types and a primary cause of cancer-associated mortality worldwide. HCC accounted for $9 \%$ of all cancer-related deaths worldwide in 2019 as per the World Health Organization report (1). Although considerable progress has been made in the diagnosis and treatment of liver cancers, the lack of good staging methods and efficient diagnostic markers results in poor prognosis (2-4). Therefore, there is an urgent requirement to identify novel molecular markers and related pathways that underlie the tumorigenesis and progression of HCC.

Recently, circular RNAs (circRNAs) have received increasing attention due to their potential function in gene regulation (5-7). circRNAs are non-coding RNAs with conserved, stable, abundant and tissue/developmental stage-specific expression $(8,9)$. circRNAs are able to mediate rolling circle translation, regulate the transcription of host genes, participate in the formation of alternatively spliced mRNAs and serve as microRNA (miRNA/miR) sponges $(8,10-12)$. circRNAs have key roles in regulating the biological development, pathogenesis and progression of numerous cancer types. Accumulating evidence has indicated that circRNAs are involved in normal physiological processes and in the development of various diseases, including liver cancer $(13,14)$. In addition, aberrant expression of certain circRNAs has been demonstrated to be related to numerous diseases, including cancer, cartilage degradation, heart disease, neurodegenerative diseases and stroke (15-17). However, only a small amount of studies examined the circRNA expression pattern in peripheral blood mononuclear cells (PBMCs) and this has not been thoroughly studied in liver cancers (18). 
Numerous studies have reported on the expression of circRNAs in cancer patients (19-21), but the findings are frequently inconsistent and no reliable biomarkers have been identified. These challenges may be attributed to several points and the key factors include individual differences in sampling and detection methods. The sample type constitutes another essential factor, as the commonly used specimens are tumor tissues. It is well known that tumor tissues are complex and contain multiple types of cells, such as immune cells, cancer cells, endothelial cells, fibroblasts and inflammatory cells $(22,23)$. This may be a major reason for the inconsistencies in previous results. Heterogeneity may be eliminated in most studies by analyzing large lumps of tumor tissues $(24,25)$. In the present study, PBMC samples were collected and examined using RNA-sequencing (RNA-seq), and differentially expressed circRNAs (DE-circRNAs) were identified. PBMCs have relatively simple components that reduce tumor heterogeneity considerably (26). In the present study, alterations in circRNA expression patterns in HCC were detected using RNA-seq. A Differential expression profile of circRNAs was identified in PBMCs from 19 clinical patients with HCC and 19 healthy controls. A total of 40 circRNAs with significant differential expression were identified. Considering that circRNA function is related to the function of the parent gene, Gene Ontology (GO) and Kyoto Encyclopedia of Genes and Genomes (KEGG) pathway analyses were performed to predict the potential functions of the DE-circRNAs in HCC. Based on the results of the sequencing, a Bioinformatics analysis and reverse transcription-quantitative (RT-q)PCR, certain functions of these DE-circRNAs in HCC were revealed. Furthermore, the potential of the selected circRNAs as biomarkers was assessed.

\section{Materials and methods}

Patients and sample collection. A total of 22 healthy individuals and 28 patients with HCC who visited Shenzhen Traditional Chinese Medicine Hospital (Shenzhen, China) from July 2018 to May 2019 were enrolled in the study and fresh blood samples were collected in EDTA tubes after obtaining written informed consent. All patients with HCC were diagnosed according to Guidelines for Diagnosis and Treatment of Primary Liver Cancer in China (2017 Edition) (27) and most of the patients received surgery or interventional therapy before blood sampling. Patients were excluded if they were pregnant or lactating, had a previous history of or currently undergoing for other malignant tumors, had cardiac disease or serious and active infections. However, patients with hepatitis $\mathrm{B}$ virus (HBV) and hepatitis $\mathrm{C}$ virus ( $\mathrm{HCV}$ ) infections were not excluded. Individuals in the control group were enrolled from The Health Examination Center of Shenzhen Traditional Chinese Medicine Hospital at random during the same period, who were selected from the Health Examination Center database with recorded medical history after completing the informed consent form. All available information and clinical characteristics of the patients with HCC are listed in Table SI. The samples of 19 controls and 19 patients with HCC were measured using RNA-seq and assayed by reverse transcription-quantitative PCR. The patients with HCC were aged $14-82$ years, with an average age of $55 \pm 18$ years, whereas the healthy controls were aged $21-75$ years, with an average age of $40 \pm 15$ years. Among the 28 patients with HCC enrolled in this study, 20 were male and 8 were female. Of the 22 controls, 17 were male and 5 were female. The study was approved by the Ethics Committee of the Shenzhen Traditional Chinese Medicine Hospital (Shenzhen, China). All experiments were performed in accordance with the relevant guidelines and regulations established by the Ethics Committee.

Processing of blood and RNA extraction. Peripheral blood ( $2 \mathrm{ml}$, with EDTA anticoagulant) was collected from patients and matched healthy controls. Ficoll-Paque PREMIUM (Cytiva) was used for the isolation of PBMCs according to the manufacturer's instructions. Total RNA was isolated using TRIzol $^{\circledR}$ (Invitrogen; Thermo Fisher Scientific, Inc.) reagent following standard procedures as previously described (28). The quality of RNA was determined by measuring the absorbance at $260 \mathrm{~nm}\left(\mathrm{~A}_{260}\right)$ and the $\mathrm{A}_{280}$ using a NanoDrop ND-1000 (Thermo Fisher Scientific, Inc.), and RNA integrity was determined based on the RNA integrity number derived from the peak area of 28s RNA, 18s RNA and 5s RNA (RIN $\geq 7$; Agilent 2100 RIN Beta Version Software; Agilent Technologies, Inc.).

RNA-seq. A total of $2 \mu \mathrm{g}$ of RNA sample from each subject was prepared as the input for RNA-seq. In the first step, ribosomal RNA (rRNA) was removed using Epicenter Ribozero ${ }^{\mathrm{TM}}$ rRNA Removal kit (Epicenter; Illumina, Inc.) and rRNA-free components were cleaned by ethanol precipitation. Next, the RNAs were submitted for library preparation according to the method described in a previous study (29). It was prepared using the VAHTS Total RNA-seq (H/M/R) Library Prep kit for Illumina (cat. no. NR603-01; Vazyme Biotech Co., Ltd.) according to the manufacturer's protocols. In brief, the following steps, including fragmentation, reverse transcription, adaptor ligation and preamplification, were performed sequentially. Finally, the libraries were purified, quality-tested and quantified using the Agilent Bioanalyzer 2100 system (Agilent Technologies, Inc.). The libraries were sequenced on Illumina HiSeq X Ten System by using HiSeq X Ten Reagent Kit (cat. no. FC-501-2501) with 2x150 bp paired-end technology.

Bioinformatics analysis. The quality of raw sequencing data in fastq format was assessed using FastQC software (v 0.11.5; https://www.bioinformatics.babraham.ac.uk/projects/download.html\#fastqc). SOAPnuke software (v2.0) was used to filter low-quality data (30). BWA software (v0.6; http://bio-bwa.sourceforge.net/bwa.shtml) was used to align the clean reads with high quality to the human reference genome (GRCh38/hg38) (31,32) with default parameters. The unaligned sequencing data were then subjected to circRNA analysis by recognition of the reverse splicing event using the CIRI2 program (33). The CIRI algorithm identified circRNA candidates using two-step filtering. First, the paired chiastic clipping signals were collected and then the false-positive junctions were removed.

Reads per million mapped reads was used for quantification of circRNA expression (34). P-values for the differences in gene expression were adjusted by false discovery rate using the Benjamini-Hochberg procedure. $\mathrm{P}<0.05$ and 
Table I. Primers for qPCR of the selected circRNAs.

\begin{tabular}{lcl}
\hline Name & Direction & Sequence $\left(5^{\prime}-3^{\prime}\right)$ \\
\hline hsa_circ_0017586 & F & GGATTTGCAAGGCTAAGTGC \\
hsa_circ_0008304 & R & CCGTAGTAAGGGTTTCGATCC \\
& F & TTTGACTTCCACAAATGCAGA \\
hsa_circ_0001181 & R & AAAAACCTTCGTCTTCAATAATTC \\
hsa_circ_0073239 & F & AACTGCCATTCAATGCACAA \\
hsa_circ_0070190 & R & GTCGGGAAGTTCAGTGGAAA \\
hsa_circ_0000369 & F & TGACTTCCGTTGGACTGATG \\
GAPDH & R & AGATGGGGTGGTTGTCACAT \\
& F & TTATGGAGGGAAACCCATCA \\
& R & TACGCACGAGGAAAACTGTG \\
& F & CAACACGGAAGTGCTGTTGT \\
\end{tabular}

F, forward; R, reverse; hsa, Homo sapiens; circ/circRNA, circular RNA; si, small interfering RNA.

$\mid \log _{2}$ (fold-change) $\mid>1$ were used as the threshold for filtering the DE-circRNAs. The R package DESeq (1.10.1) was used to analyze the differential circRNA expression. Functional annotation and pathway enrichment of the parent genes of the DE-circRNAs were performed using KEGG Orthology-based annotation system (KOBAS 3.0) (35). GO and KEGG annotation and enrichment were also performed for the parent genes and corresponding target genes of circRNAs using the KOBAS software (corrected P-value <0.05) (36-38).

To investigate the correlation between circRNAs and mRNAs in patients with HCC, interactions among the DE-circRNAs were studied and the location of the related parent genes on exons and their corresponding target miRNAs were identified. TargetScan (version no 7.1; http://www.targetscan. org/vert_71/) and miRanda (v3.3a; http://www.microrna. org/microrna/home.do) were used to predict the target miRNAs of these circRNAs. Cytoscape (v3.8.0; https://cytoscape.org/) was used to illustrate the co-expression network $(39,40)$.

$R T$ - $q P C R$. Total RNA extracted from PBMCs and cell lines was subjected to RT-qPCR validation. Specific divergent primers spanning the back-splice junction sites of the circRNAs were designed. To detect the expression levels of the selected circRNAs, cDNA was synthesized using the PrimeScript ${ }^{\mathrm{TM}}$ RT reagent kit (Takara Biotechnology Co., Ltd.) by incubating at $37^{\circ} \mathrm{C}$ for $15 \mathrm{~min}$ and then $85^{\circ} \mathrm{C}$ for $5 \mathrm{sec}$ to terminate the reaction, with a final volume of $20 \mu \mathrm{l}$. TB Green Premix Ex Taq $^{\mathrm{TM}}$ II (Takara Biotechnology Co., Ltd.) was used for qPCR analyses on ABI 7500 thermal cycler according to the manual instruction (Thermo Fisher Scientific, Inc.). After predenaturing at $95^{\circ} \mathrm{C}$ for $2 \mathrm{~min}$, the reaction system were submitted to $94^{\circ} \mathrm{C}$ for $15 \mathrm{sec}$ and $60^{\circ} \mathrm{C}, 45 \mathrm{sec}$, for 40 cycles. The internal control gene GAPDH was measured at the same time. The expression of different circRNAs was compared using the $2^{-\Delta \Delta \mathrm{Cq}}$ method (41). The primers (synthesized by Sangon Biotech) used in the present study are listed in Table I.
Cell culture and transfection. To further explore the potential diagnostic roles of the DE-circRNAs in liver cancer, the human liver cancer cell line HepG2 was obtained from the Cell Bank of the Chinese Academy of Sciences. The cell line was authenticated via short tandem repeat testing by the Chinese Academy of Sciences.

Cells were cultured in DMEM (Corning, Inc.) supplemented with $10 \%(\mathrm{v} / \mathrm{v})$ fetal bovine serum (Biological Industries) at $37^{\circ} \mathrm{C}$ in a humidified atmosphere with $5 \% \mathrm{CO}_{2}$.

Following confirmation by RT-qPCR, Homo sapiens (hsa) circ_0001181 was selected for further study. To knockdown the expression of endogenous hsa_circ_0001181 in HepG2 cells, the hsa_circ_0001181 siRNA (corresponding double stranded oligo sequences are as follows, sense, 5'-CACCGA AGCTGGTTGATGATAATTCGAAAATTATCATCAACC AGC-3'; antisense, 5'-AAAAGCTGGTTGATGATAATT TTCGAATTATCATCAACCAGCTTC-3') was packaged with a pGLV2-U6 lentivector packaging plasmid mix (Sangon Biotech Co., Ltd.) in a $293 \mathrm{~T}$ packaged cell line (cat. no. CRL-11268; ATCC). The routine culture conditions are as follows: DMEM $+10 \% \mathrm{FBS}$ at $37^{\circ} \mathrm{C}$ and $5 \% \mathrm{CO}_{2}$. When packaging, one tube contained $20 \mu \mathrm{g}$ lentiviral plasmid, $15 \mu \mathrm{g}$ packaging vector (pCMV-dR8.9; Addgene, Inc.) and $10 \mu \mathrm{g}$ envelope (pCMV-VSV-G; Addgene, Inc.) were prepared, then $500 \mu 1$ Trans-EZ solutions (Sangon Biotech Co., Ltd.) was diluted with $4.5 \mathrm{ml}$ Opti-MEM medium (cat. no. 31985; Gibco; Thermo Fisher Scientific, Inc.). The Trans-EZ solution was then added into the plasmid mix to obtain the transfection complex, it was incubated $20 \mathrm{~min}$ at room temperature; the mixture was added to $293 \mathrm{~T}$ cell line and cultured for about $48 \mathrm{~h}$ at $37^{\circ} \mathrm{C}$ and $5 \% \mathrm{CO}_{2}$. The lentiviral particles were isolated by ultracentrifugation $\left(4^{\circ} \mathrm{C}, 82700 \mathrm{x}\right.$ g and $\left.2 \mathrm{~h}\right)$. An empty vector was transfected as the negative controls. HepG2 cells were then transfected with the lentiviral vector particles (MOI=8). After incubation at $37^{\circ} \mathrm{C}$ for $24-36 \mathrm{~h}$, RT-qPCR was also performed to confirm the knockdown efficiency. 
A

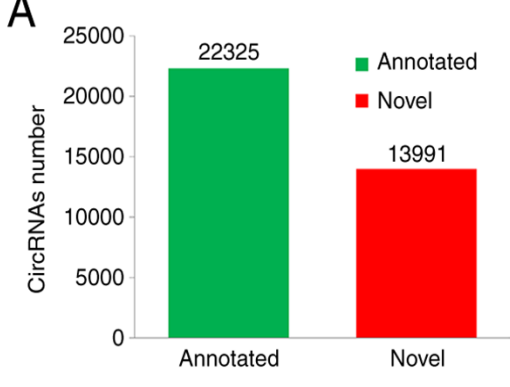

B

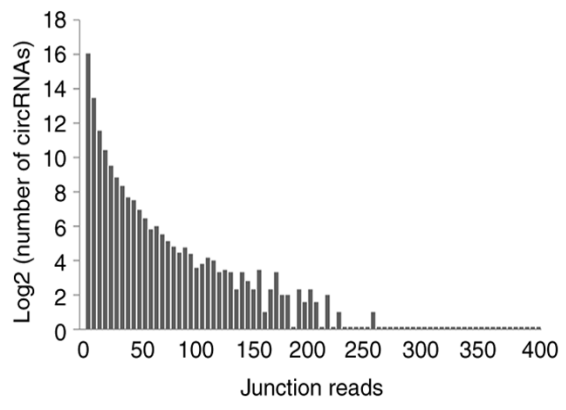

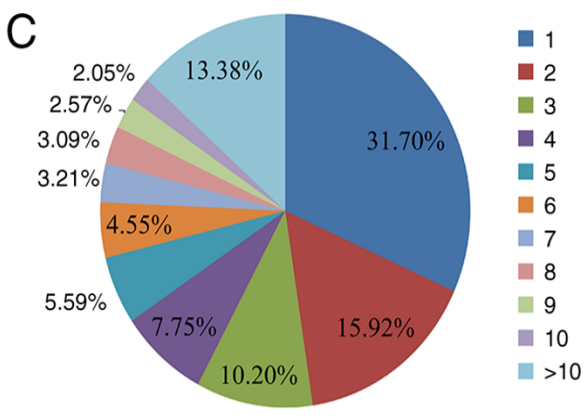

F

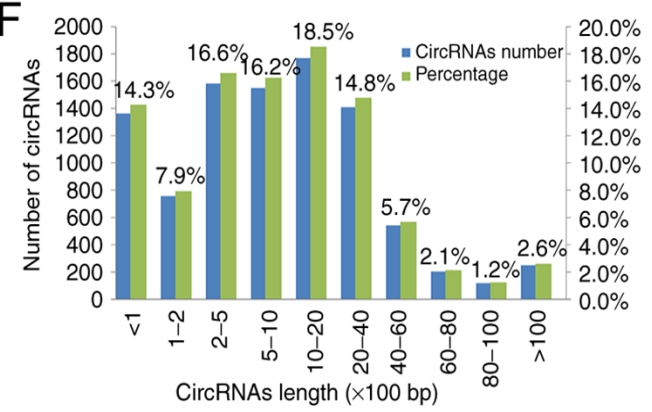

C

2

3

5

6

7

9
D

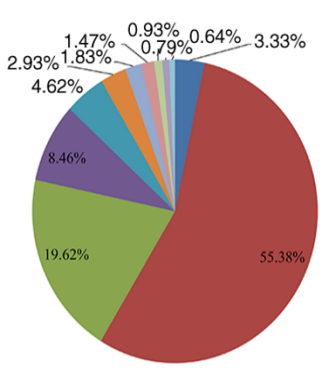

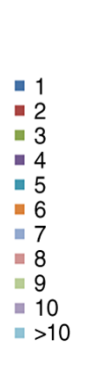

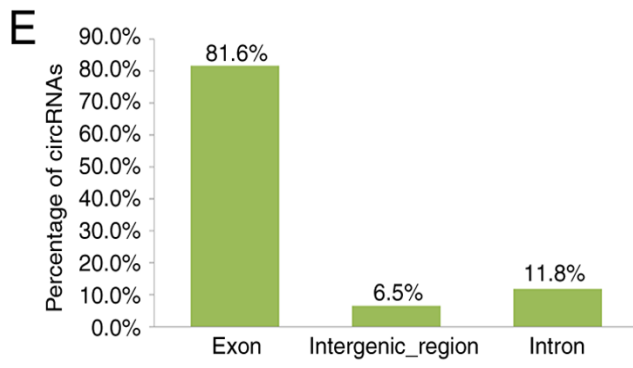

Figure 1. Characterization of the circRNAs sequenced in PBMCs of patients with HCC and normal controls. (A) Comparison of circRNAs identified in the present study and with circBase. (B) The number of circRNAs and back-spliced reads identified in PBMCs of patients with HCC and normal controls. (C) Number of circRNAs produced from one gene. (D) Exon number of identified circRNAs. (E) Distribution of all the circRNAs identified in the present study. (F) Length distribution of circRNAs detected. CircRNA, circular RNA; HCC, hepatocellular carcinoma; PBMC, peripheral blood mononuclear cells.

Proliferation assay. Proliferation of the transfected cells was measured using a Cell Counting Kit-8 (CCK-8; Dojindo Molecular Technologies, Inc.) according to the manufacturer's protocol. In total, $\sim 5,000$ cells were seeded into each well and after incubation at $37^{\circ} \mathrm{C}$ for 24,48 or $72 \mathrm{~h}$ in a 96 -well microplate (Corning, Inc.), CCK-8 stain (5 $\mu \mathrm{l})$ and $100 \mu \mathrm{l}$ of fresh medium were added to each well, followed by incubation at $37^{\circ} \mathrm{C}$ for $2 \mathrm{~h}$. The absorbance was measured at $450 \mathrm{~nm}$ using a Multiskan FC microplate reader (Thermo Fisher Scientific, Inc.). HepG2 cells without any transfection served as the blank control and cells transfected with the empty vector were used as the negative control (NC) (42).

Statistical analysis. Data were analyzed using SPSS software (version 18.0; SPSS, Inc.) and graphs were generated using GraphPad Prism 8.0 (GraphPad Software, Inc.). Comparisons between the HCC and control groups were performed using Student's t-test. The results of the cell proliferation assay were compared between different groups by one-way ANOVA using Tukey's test. RT-qPCR data are presented as the mean \pm standard deviation of at least three independent tests. Statistical significance was set at $\mathrm{P}<0.05$. Receiver operating characteristic (ROC) curve analysis was performed to evaluate the diagnostic accuracy of the different genes analyzed.

\section{Results}

CircRNA profiling of PBMCs from patients with HCC and normal controls. A total of $1,807.83 \mathrm{M}$ raw reads with a mean length of $300 \mathrm{bp}$ was obtained. Quality control analysis indicated that 93.64-95.19\% of the sequencing data had a $\mathrm{Q}$ score of $>30$. The mean GC content of the clean reads for PBMCs from HCCs and controls ranged from 45.02 to $47.99 \%$.
Due to the high quality of the sequencing data obtained, the present results are reliable.

A total of 36,316 unique circRNAs were sequenced from the PBMC samples by counting the junction reads (count number $>2$ ) and a total of 2,239-9,897 circRNAs were identified from these 38 PBMC samples. Of these, 22,325 circRNAs were already listed in the circBase that were identified in previous studies and 13,991 novel circRNAs were identified in the present study. The host genes of the identified circRNAs were distributed across all chromosomes. Most of them contained two or more unique junction reads (Fig. 1A and B).

Multiple circRNAs may be generated from one gene as a result of the alternative back-splicing mechanism (43). In the present study, it was analyzed whether alternative back-splicing is related to circRNA diversity in PBMCs from patients with HCC. As presented in Fig. 1C, two or more circRNAs were produced from $68.3 \%$ of the host genes. Of note, $>10$ circRNAs were generated from certain genes. Most of the circRNAs were identified from the exonic regions and consisted of 2-5 exons. Among them, the circRNAs consisted of 2, 3, 4, 5 and 6 exons accounted for 55.38, 19.62, 8.46, 4.62 and $2.93 \%$ respectively (Fig. 1D and E). In addition, the lengths of most $71.8 \%$ circRNAs ranged between 200 and 6,000 nucleotides (Fig. 1F).

Detection of dysregulated circRNAs in PBMCs from patients with HCC. Among the circRNAs identified, only 608 and 474 circRNAs were co-expressed in all control and HCC samples, respectively. Of the co-expressed circRNAs, 27 upregulated and 13 downregulated circRNAs were determined to be differentially expressed between the HCC and healthy controls with a fold-change of $\geq 2.0$ and $\mathrm{P}<0.05$ by scatter plot filtering (Fig. 2A and Table II). Hierarchical clustering analysis of the 
Table II. Differentially expressed of circRNAs in peripheral blood mononuclear cells from patients with hepatocellular carcinoma.

\begin{tabular}{|c|c|c|c|c|c|}
\hline CircRNA & Circbase ID & $\begin{array}{l}\text { Host gene } \\
\text { symbol }\end{array}$ & $\begin{array}{l}\text { Direction of } \\
\text { regulation }\end{array}$ & Adj P-value & Location \\
\hline CHR6:31238850|31323369 & Novel & HLA-C & Up & $3.91617 \times 10^{-12}$ & $\mathrm{n} / \mathrm{a}$ \\
\hline CHR13:50571210150618904 & hsa_circ_0100694 & $\mathrm{n} / \mathrm{a}$ & Up & $8.68089 \times 10^{-5}$ & Intergenic region \\
\hline CHR7:128218007|128278951 & hsa_circ_0133256 & $\mathrm{n} / \mathrm{a}$ & Up & $8.68089 \times 10^{-5}$ & Intergenic region \\
\hline CHR10:581580515842668 & hsa_circ_0017586 & GDI2 & Up & 0.000268902 & Exon \\
\hline CHR3:141811903|141820683 & hsa_circ_0008304 & TFDP2 & Down & 0.000327428 & Exon \\
\hline CHR21:30693542I30702014 & hsa_circ_0001181 & BACH1 & Up & 0.000359788 & Exon \\
\hline CHR5:82832826182850857 & hsa_circ_0073239 & VCAN & Up & 0.000409673 & Exon \\
\hline CHR4:79747191179772210 & hsa_circ_0070190 & BMP2K & Up & 0.000745549 & Exon \\
\hline CHR11:128628010|128642880 & hsa_circ_0000369 & FLI1 & Up & 0.000950703 & Exon \\
\hline CHR10:28408580|28420620 & hsa_circ_0093467 & MPP7 & Up & 0.000987788 & Exon \\
\hline CHR8:22332467I22333137 & hsa_circ_0083619 & PPP3CC & Down & 0.00124762 & Exon \\
\hline CHR9:35546427I35548532 & hsa_circ_0002702 & RUSC2 & Up & 0.002681018 & Exon \\
\hline CHR16:19627436l19659204 & hsa_circ_0005699 & VPS35L & Up & 0.002681018 & Exon \\
\hline CHR2:228356263I228389631 & hsa_circ_0058514 & AGFG1 & Up & 0.002889102 & Exon \\
\hline CHR12:10530731I10531304 & Novel & KLRK1 & Down & 0.002895133 & Exon \\
\hline CHR5:82815168I82818128 & hsa_circ_0073239 & VCAN & Up & 0.003273089 & Exon \\
\hline CHR4:17963526117974508 & hsa_circ_0069285 & LCORL & Down & 0.003518927 & Exon \\
\hline CHR13:21732061I21746820 & hsa_circ_0007547 & SKA3 & Up & 0.004447874 & Exon \\
\hline CHR12:66597491|66611015 & hsa_circ_0005505 & IRAK3 & Up & 0.005338426 & Exon \\
\hline CHR5:82832826182838087 & hsa_circ_0073239 & VCAN & Up & 0.005492673 & Exon \\
\hline CHR9:99271955|99327765 & hsa_circ_0087636 & CDC14B & Up & 0.005492673 & Exon \\
\hline CHR5:150838349|150859050 & hsa_circ_0074623 & SLC36A1 & Up & 0.005492673 & Exon \\
\hline CHR1:211526581|211527809 & hsa_circ_0007234 & TRAF5 & Down & 0.005492673 & Exon \\
\hline CHR4:146767108I146770713 & hsa_circ_0003187 & ZNF827 & Down & 0.005492673 & Exon \\
\hline CHR6:145992375I146007432 & hsa_circ_0130925 & EPM2A & Down & 0.005492673 & Exon \\
\hline CHR5:130590788I130591885 & Novel & CDC42SE2 & Down & 0.005492673 & Intron \\
\hline CHR13:95686859|95715113 & hsa_circ_0008463 & ABCC4 & Up & 0.005715187 & Exon \\
\hline CHR11:108200941I108202764 & hsa_circ_0024235 & ATM & Down & 0.005715187 & Exon \\
\hline CHR18:2890559|2892484 & hsa_circ_0004658 & EMILIN2 & Up & 0.006202165 & Exon \\
\hline CHR2:174819601I174820960 & hsa_circ_0002642 & SP3 & Up & 0.006497297 & Exon \\
\hline CHR1:46032241|46032692 & hsa_circ_0000067 & AKR1A1 & Down & 0.006946213 & Exon \\
\hline CHR9:117116916l117117565 & Novel & AKNA & Down & 0.006977218 & Intron \\
\hline CHR11:129979324I129992408 & hsa_circ_0000372 & APLP2 & Up & 0.007668903 & Exon \\
\hline CHR20:57574398I57574864 & Novel & CTSZ & Up & 0.007894677 & Intron \\
\hline CHR7:27668990|27672064 & hsa_circ_0003958 & HIBADH & Down & 0.007894677 & Exon \\
\hline CHR8:67484717l67485741 & hsa_circ_0136924 & MYBL1 & Down & 0.007894677 & Exon \\
\hline CHR10:75830428175834661 & hsa_circ_0018881 & VCL & Up & 0.008421014 & Exon \\
\hline CHR14:91947920|91957146 & hsa_circ_0003045 & PPP4R3A & Up & 0.00909621 & Exon \\
\hline CHR3:150834125I150845771 & hsa_circ_0067735 & MED12L & Up & 0.009420242 & Exon \\
\hline CHR15:50923614I50941082 & hsa_circ_0035249 & TRPM7 & $\mathrm{Up}$ & 0.009420242 & Exon \\
\hline
\end{tabular}

hsa, Homo sapiens; circ/circRNA, circular RNA; n/a, not available; CHR, chromosome; Adj, adjusted.

abnormally expressed circRNAs suggested that the circRNA expression pattern was significantly different between patients with HCC and healthy controls (Fig. 2B).

The significantly dysregulated mRNAs are presented in the heatmap of the inter-sample correlation (Pearson's correlation coefficient). As indicated in Fig. 2C, the circRNA expression levels were significantly different between the HCC and control groups, whereas a slight variation was observed within each group.

Construction of the circRNA-miRNA-mRNA interaction network. circRNAs inhibit miRNA-mediated gene regulation by serving as miRNA sponges (44). The potential target miRNAs of HCC-specific circRNAs were identified using 

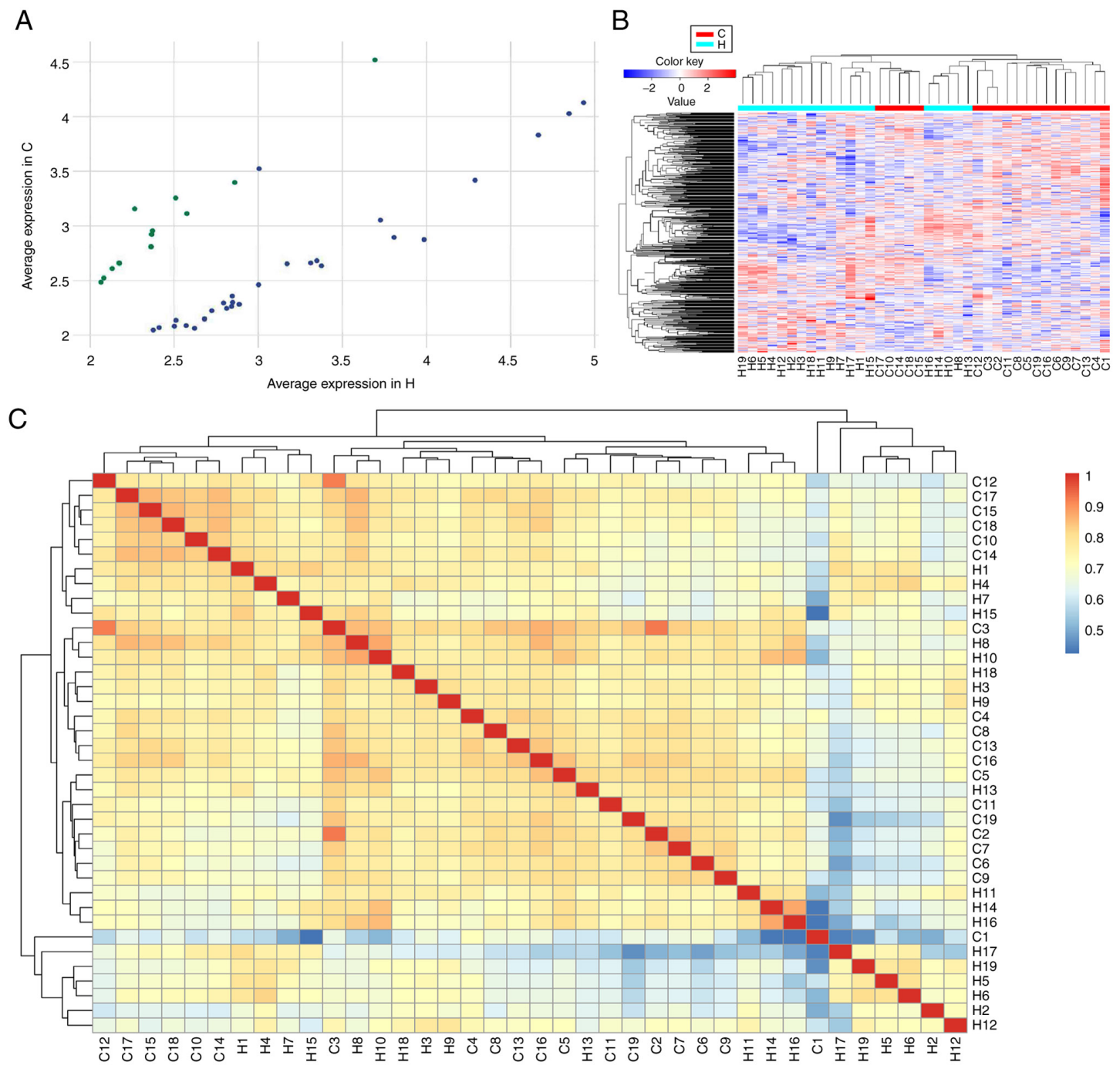

Figure 2. Differentially expressed circRNAs in patients with HCC and normal controls. (A) Scatter plot of the difference in circRNA expression between the HCC and healthy controls. (B) Hierarchical clustering results showed the circRNA expression patterns of the HCC and control groups. (C) Heatmap indicating the inter-sample correlation (Pearson's correlation coefficient) of these significantly dysregulated circRNAs. CircRNA, circular RNA; HCC, hepatocellular carcinoma; C, controls; $\mathrm{H}$, patients with HCC.

miRanda. Cytoscape was used to generate a circRNA-miRNA interaction network through the predicted miRNAs and circRNAs $(39,40)$. All abnormally expressed circRNAs were predicted based on complementary sequences of miRNAs. A total of 514 miRNAs that are able to bind to circRNAs were identified. The complete network of 11 DE-circRNAs (circular nodes) and target miRNA (triangular nodes) interactions are delineated in Fig. 3A, where different colors of the miRNAs presented in the figure indicate differential expression in HCC compared with normal controls by miRNA sequencing. Increasing expression levels of the miRNAs is represented as changes in color increased from green to blue to red, as indicated in the legend bar on the top right corner of the figure.

To study the potential functions of the circRNAs, a regulatory network of circRNA-miRNA-mRNA was generated based on these circRNAs. Fig. 3B indicates the top 150 circRNA-miRNA-mRNA interactions with significant P-values $(\mathrm{P}<0.05)$. The results suggested that a single circRNA is able to interact with several miRNAs by acting as an antagonist. For instance, $>50$ miRNAs were predicted to bind to hsa_circ_0070190 and hsa_circ_0001181. Furthermore, both these circRNAs contained binding sites for hsa-miR-4659b-3p, hsa-miR-3614-3p and hsa-miR-4659a-3p. Different circRNAs with the same miRNA binding site may interact with one miRNA and regulate the expression level of a target gene in HCC. As presented in Fig. 3C, miRNA/mRNA interactions were predicted using the miRNA database (Fig. 3C).

As shown in Fig. 3D, functional annotation indicated that these abnormally expressed circRNAs have important roles in 'nucleoplasm', 'cytoplasm' and 'nucleus' of cellular components; 'positive regulation of transcription from RNA polymerase II promoter', 'negative regulation of transcription from RNA polymerase II promoter' and 'positive regulation of cell proliferation' of biological processes and molecular functions, including 'protein binding', 'transcription factor binding' and 'protein kinase binding'. It was indicated that the target mRNAs were involved in cancer-related pathways, including the TNF, MAPK and the PI3K-AKT signaling pathways, which were implicated in carcinogenesis and the cell cycle (Fig. 3E). In addition, several pathways associated with HCC were found, including pathways in cancer, TNF signaling pathway, hepatitis B, hepatitis C and hepatocyte differentiation (Fig. 3E). 

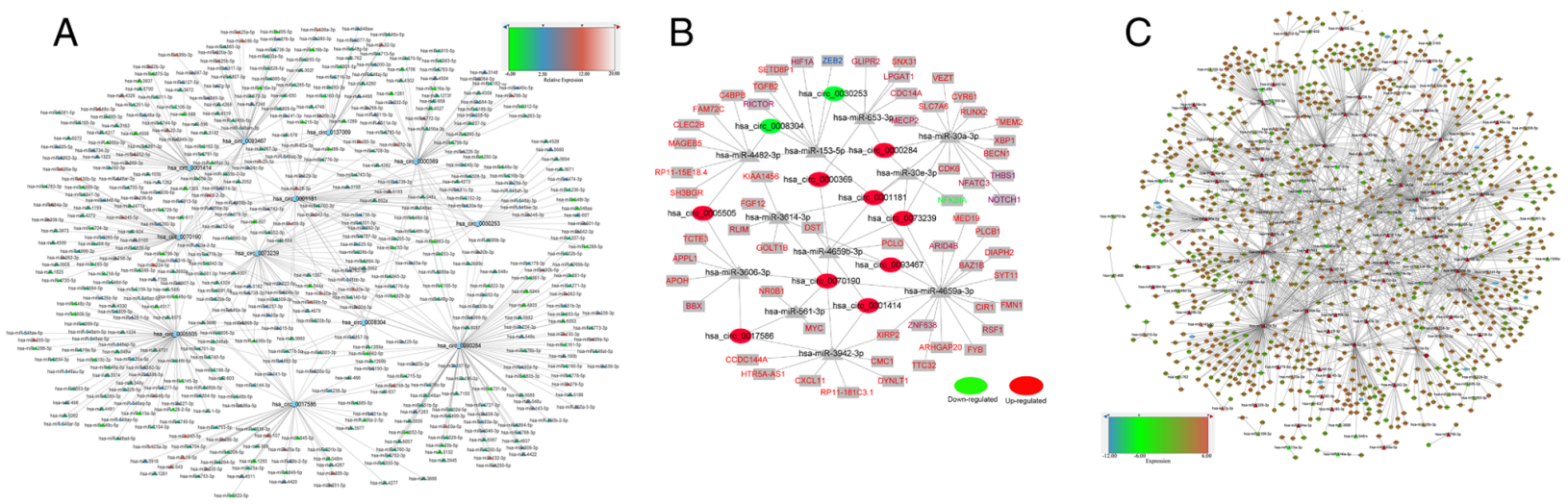

$\mathrm{D}$
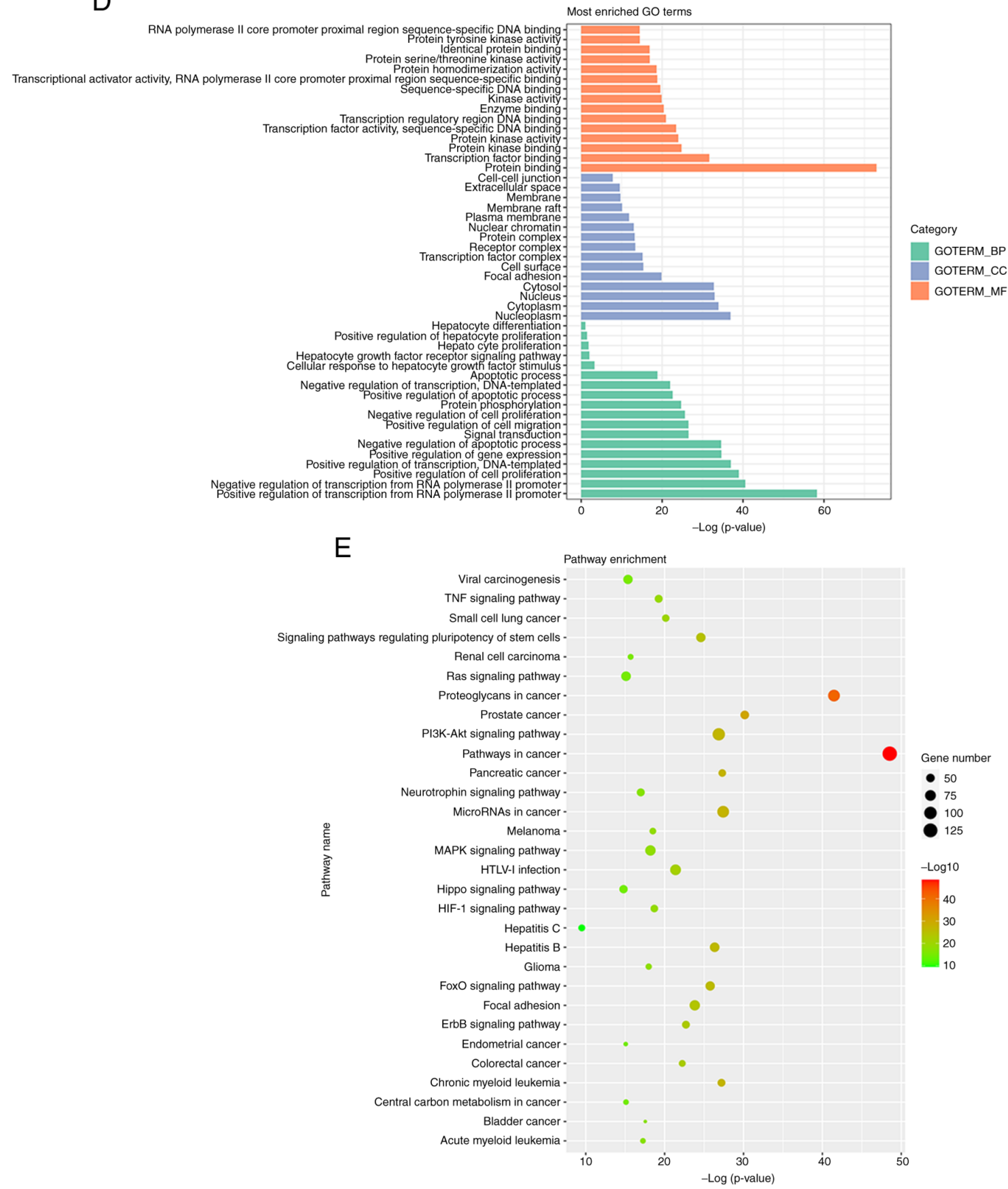

Figure 3. Construction of the circRNA-miRNA-mRNA competing endogenous RNA regulatory networks. (A) CircRNA-miRNA network of the interactions between circRNAs and its target miRNAs. (B) CircRNA-miRNA-mRNA network constructed from the top-11 circRNAs and their targets based on sequence-pairing prediction. (C) Networks of the interactions of the target miRNAs and their predicted genes. (D) Enriched GO terms. (E) Enriched KEGG pathways from the circRNA-miRNA-mRNA network. CircRNA, circular RNA; miRNA, microRNA; hsa, Homo sapiens; GO, Gene Ontology; KEGG, Kyoto Encyclopedia of Genes and Genomes; BP, biological process; CC, cellular component; MF, molecular function. 

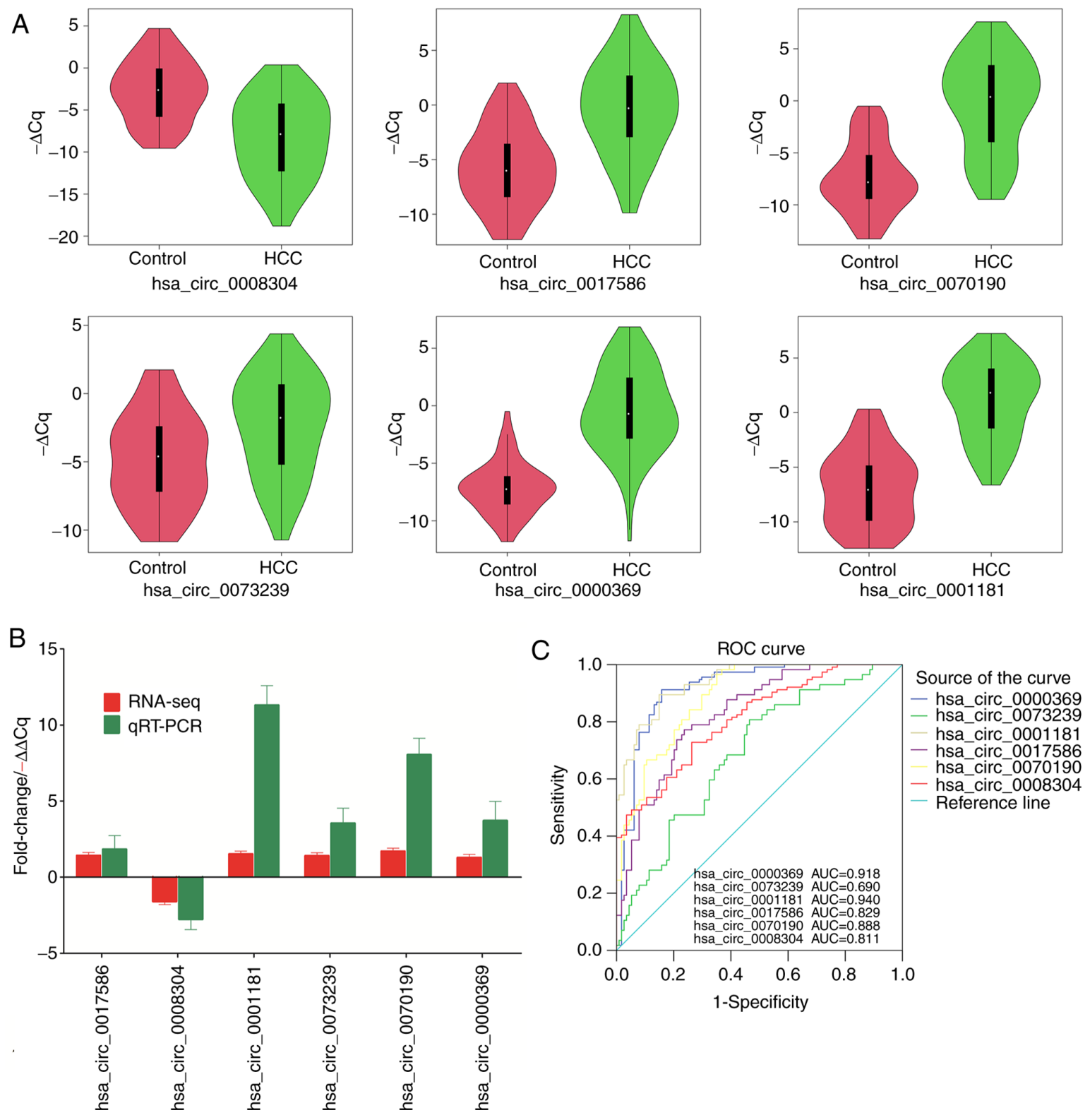

Figure 4. Validation of the expression of significant transcripts by RT-qPCR and ROC analysis of the circRNAs. (A) Violin plots displaying the expression levels of the six circRNAs measured by RT-qPCR. Significant differences were present between HCC and healthy controls (P<0.001). (B) Comparison of RNA-seq results and RT-qPCR results in the six circRNAs. (C) ROC curve analysis of hsa_circ_0017586, hsa_circ_0008304, hsa_circ_0001181, hsa_circ_0073239, hsa_circ_0070190 and hsa_circ_0000369. AUC values are provided on the graphs. ROC, receiver operating characteristic; AUC, area under the ROC curve; RT-qPCR, reverse transcription-quantitative PCR; circRNA, circular RNA; hsa, Homo sapiens; HCC, hepatocellular carcinoma; Cq, quantification threshold; Fold-change, the ratio of normalized sequencing counts between HCC group and control group; RT-qPCR, reverse transcription-quantitative PCR.

Validation of the DE-circRNAs. To verify the sequencing and prediction results, the expression levels of the top six circRNAs annotated in circBase were analyzed, including five upregulated and one downregulated circRNA from the 28 clinical cases with HCC and 22 healthy controls by RT-qPCR. GAPDH was used as an internal control. The results indicated that the relative expression levels of hsa_circ_0017586, hsa_circ_0001181, hsa_circ_0073239, hsa_circ_0070190 and hsa_circ_0000369 in PBMCs from patients with HCC were significantly higher compared with those in healthy controls, whereas hsa_circ_0008304 was downregulated in patients with HCC compared with that in the controls (Fig. 4A). These results are consistent with the results of the RNA-seq analysis (Fig. 4B).
ROC curve analysis was performed to evaluate the potential of the circRNAs as diagnostic biomarkers in all 50 samples (28 HCC and 22 controls) collected for the present study. As presented in Fig. 4C, the area under the ROC curve to differentiate patients with $\mathrm{HCC}$ from healthy controls was 0.690 (95\% CI: 0.622-0.758) for hsa_circ_0073239, 0.888 (95\% CI: 0.847-0.928) for hsa_circ_0070190, 0.918 (95\% CI: 0.881-0.956) for hsa_circ_0000369, 0.811 (95\% CI: 0.757-0.865) for hsa_circ_0008304, 0.829 (95\% CI: 0.777-0.881) for hsa_circ_0017586 and 0.940 (95\% CI: 0.913-0.967) for hsa_circ_0001181, indicating the potential of these circRNAs as diagnostic markers for HCC. Among them, hsa_circ_0001181 and hsa_circ_0000369 had the highest potential as diagnostic markers and may be used for diagnosing HCC. 
A

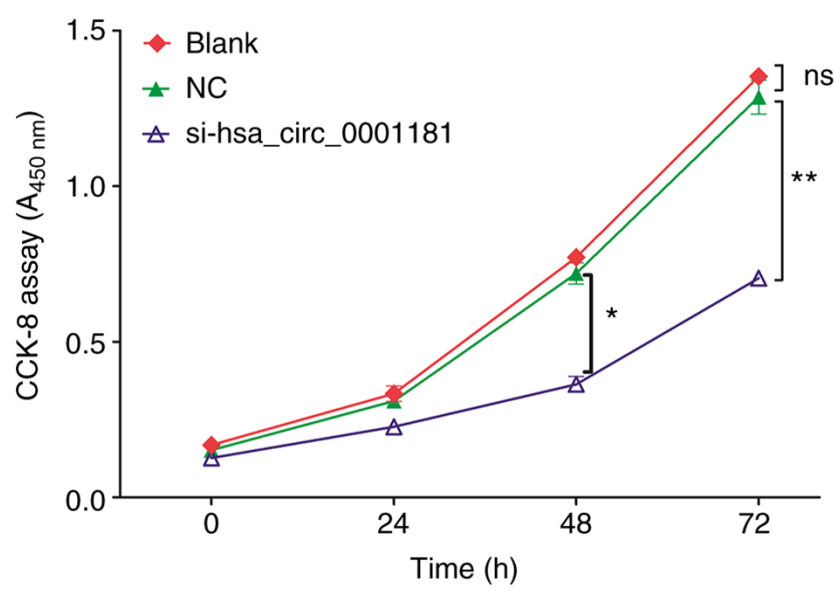

B

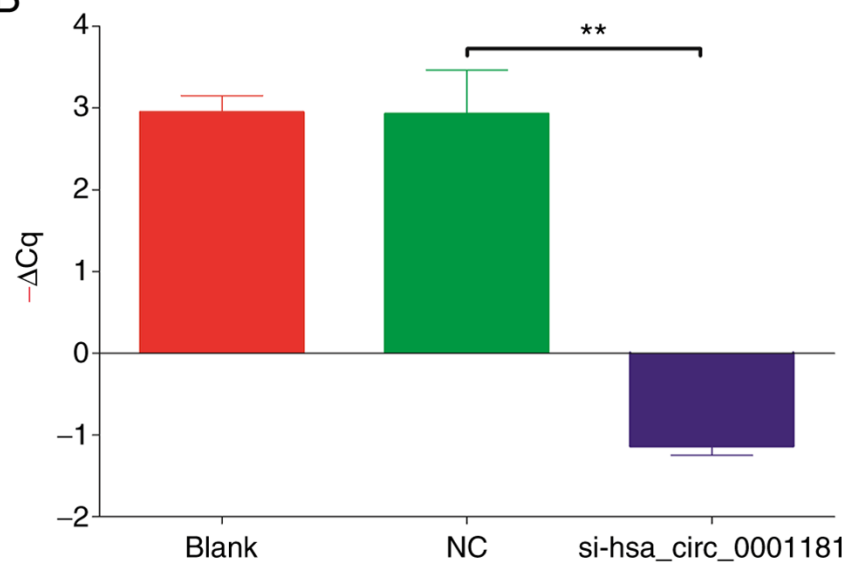

Figure 5. (A) The proliferation of HepG2 cells was evaluated using a CCK-8 assay with transfection of si-hsa_circ_0001181 after 0, 24,48 and 72 h. The A 450 nm was measured to reflect the amount of viable cells. The cell proliferation level of the si-hsa_circ_0001181 group was significantly lower than that of the blank or NC group after $48 \mathrm{~h}$ and highly significantly lower after $72 \mathrm{~h}$. However, the blank group and NC group exhibited no significant difference. (B) Reverse transcription-quantitative PCR detection of the circRNA knockdown efficiency, $-\Delta \mathrm{Cq}$ was used to denote the expression level. ${ }^{*} \mathrm{P}<0.05$, $^{* *} \mathrm{P}<0.01$. ns, no significance; $\mathrm{A}_{450 \mathrm{~nm}}$, absorbance at $450 \mathrm{~nm}$; NC, negative control; si-hsa_circ_0001181, small inhibitory RNA targeting Homo sapiens circRNA 0001181; circRNA, circular RNA; $-\Delta \mathrm{Cq}$, quantification threshold.

circRNAs may be involved in the regulation of HCC cell growth. The results of the CCK-8 proliferation assay (Fig. 5A) were analyzed by ANOVA. It was indicated that significant differences were present between different groups and different times $(\mathrm{P}<0.001)$, suggesting that the knockdown of hsa_circ_0001181 expression in HepG2 cells significantly reduced cell growth compared to the blank (no transfection) and NC (transfected with empty pGLV2-U6 lentivector) groups, particularly at 48 and $72 \mathrm{~h}$. There was no significant difference between the blank and NC groups $(\mathrm{P}=0.056)$, suggesting that cell proliferation was specifically affected by hsa_circ_0001181 and not by the vector. RT-qPCR was performed to confirm the knockdown efficiency and the results indicated that the expression level of hsa_circ_0001181 was significantly downregulated following hsa_circ_0001181 silencing (Fig. 5B).

\section{Discussion}

CircRNAs were once thought of as by-products of aberrant RNA splicing, but with the development of RNA-seq and bioinformatics technologies in the past few decades, a number of circRNAs have been functionally characterized, revealing their ability to adsorb to miRNA (45) and regulate the expression of the target gene(s) (46). Studies have also indicated that circRNAs may be translated into functional proteins (47). In addition, studies have revealed that circRNAs are highly abundant, stable and resistant to exonuclease $(8,48)$. Furthermore, differences in circRNAs exist among different tissues and diseases $(49,50)$. Thus, circRNAs have demonstrated great potential for application in early diagnosis and as therapeutic targets, and it is worthwhile investigating their mechanistic involvement in disease occurrence and development.

Several studies on cancers have focused on PBMCs (51-53). Certain studies have also reported an association with immunological diseases. PBMCs consist of monocytes and lymphocytes, which originate from the innate and adaptive immune systems. Changes in gene expression in PBMCs are generally associated with the status of tumors and tumor-related immune diseases (54), suggesting that the DE-circRNAs in PBMCs may be important potential biomarkers for the diagnosis and prognosis of liver diseases.

In the present study, circRNAs were identified in PBMCs from 19 patients with HCC and 19 controls using RNA-seq. Profiling of the DE-circRNAs led to the identification of 40 DE-circRNAs between HCC and healthy controls. Among them, 27 were upregulated and 13 were downregulated and of these, certain selected DE-circRNAs were confirmed by RT-qPCR. For the ROC analysis, 50 PBMCs samples were involved, which includes 38 samples were used for sequencing and another 12 PBMCs samples collected. It is a limitation of the present study that there was no separate cohort for RT-qPCR validation and further patients with HCC will be collected for future study. Among the DE-circRNAs, hsa_circ_0001181 and hsa_circ_000369 demonstrated a high diagnostic potential in patients with $\mathrm{HCC}$ and represent potential diagnostic biomarkers for HCC prediction. A CCK-8 assay was performed and the results indicated that silencing of hsa_circ_0001181 reduced the proliferation of HepG2 cells. This suggests that circRNA 0001181 may be involved in the genesis and development of HCC and may thus serve as a potential therapeutic target for HCC.

To investigate the potential function of these circRNAs profiled, a circRNA-miRNA-mRNA interaction network was constructed. Most of the circRNAs were newly identified to be related to HCC. As one of the types of competing endogenous RNAs, circRNAs have important roles in regulating gene expression through sequestering miRNAs. Numerous miRNAs in the network were studied previously, such as hsa-miR-30a-3p, which had been reported to be related to numerous cancer types, including lung (55), breast (56), prostatic (57) and pancreatic cancer (58). It has also been reported to be related 
to hepatic astrocyte fibrosis and autophagy (59). It is the target of hsa-circ-0001181, hsa-circ-0073239 and hsa-circ-0000284, which were determined to be significantly dysregulated in HCC in the present study. It is inferred that these circRNAs may have important roles in the genesis and development of tumors in numerous cancer types.

In the present study, $>100,000$ circRNAs were profiled from PBMCs of patients with HCC and only those with counts of $>2$ were considered to be reliable circRNAs for the follow-up experiments. Differential expression analysis was performed only for these co-expressed circRNAs and this may be the main reason why only 40 DE-circRNAs were identified in the present study.

Although only a limited number of DE-circRNAs were identified in the present study, most of them were detected for the first time in patients with HCC and they were confirmed by RT-qPCR. Furthermore, GO functional annotation results indicated that these DE-circRNAs were mainly enriched in protein binding, regulation of cell proliferation, signal transduction, protein phosphorylation, hepatocyte differentiation, among others, and were mainly involved in cancer-associated pathways related to HCC. These included pathways in cancer, the TNF signaling pathway, ErbB signaling pathway, MAPK signaling pathway, hepatitis B, hepatitis C and hepatocyte differentiation, which indicates that the host genes of these circRNAs may participate in the regulation of HCC development through related molecular functions or signaling pathways. Ni et al (60) reported that lncRNA inhibits HCC progression through CUL4A-mediated LATS1 ubiquitination and increased YAPS127 phosphorylation. Wang et al (61) indicated that SIRT4 inhibits glutamine metabolism and increases the level of adenosine diphosphate/adenosine monophosphate (AMP) and blocks the mTOR signaling pathway through phosphorylation of AMP kinase $\alpha$ that has an antitumor effect in HCC.

In the present study, circRNA expression patterns in PBMCs from HCC were determined by RNA-seq, indicating that patients with HCC had distinct circRNA expression signatures when compared to healthy controls. ROC analysis indicated that hsa_circ_0073239, hsa_circ_007090, hsa_circ_0008304, hsa_circ_0017586,hsa_circ_0001181 and hsa_circ_0000369 may potentially be used as biomarkers for HCC diagnosis. In addition, silencing of hsa_circ_0001181 reduced the growth of HepG2 cells, suggestive of its potential therapeutic target of HCC.

\section{Acknowledgements}

Not applicable.

\section{Funding}

This research was funded by the Shenzhen Science and Technology Project and Sanming Project of Medicine in Shenzhen (grant nos. JCYJ20170817094901026, JCYJ20180302173542393 and SZSM201612074).

\section{Availability of data and materials}

The datasets generated and/or analyzed during the current study are available in the NCBI project repository (accession no. PRJNA754685; https://dataview.ncbi.nlm.nih.gov/object/ PRJNA754685?reviewer=ofg2bodnrahhf5de9utin5g22k).

\section{Authors' contributions}

$\mathrm{XZ}$ and $\mathrm{ZH}$ contributed to the conception, design, performing of experiments and analysis of the data. XS, RH and WM contributed to sample collection and cell culture. WZ, SX, BZ, LZ and QL contributed to sample collection and CCK-8 assay. QG, LZ and ZH contributed to the bioinformatics analysis. WF and RH contributed to the acquisition of data and cell culture. ZH and WF confirm the authenticity of all the raw data. All authors read and approved the final manuscript.

\section{Ethics approval and consent to participate}

The experimental protocol was established, according to the ethical guidelines of the Helsinki Declaration and was approved by the Human Ethics Committee of Shenzhen Traditional Chinese Medicine Hospital (Shenzhen, China). Written informed consent was obtained from individual or guardian participants.

\section{Patient consent for publication}

Not applicable.

\section{Competing interests}

The authors declare that they have no competing interests.

\section{References}

1. Balogh J, Victor D III, Asham EH, Burroughs SG, Boktour M, Saharia A, Li X, Ghobrial RM and Monsour HP Jr: Hepatocellular carcinoma: A review. J Hepatocell Carcinoma 3: 41-53, 2016.

2. Zhang Y, Li T, Guo P, Kang J, Wei Q, Jia X, Zhao W, Huai W, Qiu Y, Sun L and Han L: MiR-424-5p reversed epithelial-mesenchymal transition of anchorage-independent HCC cells by directly targeting ICAT and suppressed HCC progression. Sci Rep 4: 6248, 2014

3. Chan KM, Kuo CF, Hsu JT, Chiou MJ, Wang YC, Wu TH, Lee CF, Wu TJ, Chou HS and Lee WC: Metformin confers risk reduction for developing hepatocellular carcinoma recurrence after liver resection. Liver Int 37: 434-441, 2017.

4. Wu TJ, Chang SS, Li CW, Hsu YH, Chen TC, Lee WC, Yeh CT and Hung MC: Severe hepatitis promotes hepatocellular carcinoma recurrence via NF-kB pathway-mediated epithelial-mesenchymal transition after resection. Clin Cancer Res 22: 1800-1812, 2016.

5. Ivanov A, Memczak S, Wyler E, Torti F, Porath HT, Orejuela MR, Piechotta M, Levanon EY, Landthaler M, Dieterich C and Rajewsky N: Analysis of intron sequences reveals hallmarks of circular RNA biogenesis in animals. Cell Rep 10: 170-177, 2015.

6. Chen LL: The biogenesis and emerging roles of circular RNAs. Nat Rev Mol Cell Biol 17: 205-211, 2016.

7. Zhang Y,Zhang XO, Chen T, Xiang JF, Yin QF, Xing YH, Zhu S, Yang $L$ and Chen LL: Circular intronic long noncoding RNAs. Mol Cell 51: 792-806, 2013.

8. Memczak S, Jens M, Elefsinioti A, Torti F, Krueger J, Rybak A, Maier L, Mackowiak SD, Gregersen LH, Munschauer M, et al: Circular RNAs are a large class of animal RNAs with regulatory potency. Nature 495: 333-338, 2013.

9. Lasda E and Parker R: Circular RNAs: Diversity of form and function. RNA 20: 1829-1842, 2014.

10. Jeck WR, Sorrentino JA, Wang K, Slevin MK, Burd CE, Liu J, Marzluff WF and Sharpless NE: Circular RNAs are abundant, conserved, and associated with ALU repeats. RNA 19: 141-157, 2013. 
11. Hansen TB, Jensen TI, Clausen BH, Bramsen JB, Finsen B, Damgaard CK and Kjems J: Natural RNA circles function as efficient microRNA sponges. Nature 495: 384-388, 2013.

12. Guo JU, Agarwal V, Guo $\mathrm{H}$ and Bartel DP: Expanded identification and characterization of mammalian circular RNAs Genome Biol 15: 409, 2014

13. Ren S, Xin Z, Xu Y, Xu J and Wang G: Construction and analysis of circular RNA molecular regulatory networks in liver cancer. Cell Cycle 16: 2204-2211, 2017.

14. Wang M, Gu B, Yao G, Li P and Wang K: Circular RNA expression profiles and the pro-tumorigenic function of circRNA_10156 in hepatitis B virus-related liver cancer. Int J Med Sci 17: 1351-1365, 2020 .

15. Zhang P, Ming Y, Ye Q and Niu Y: Comprehensive circRNA expression profile during ischemic postconditioning attenuating hepatic ischemia/reperfusion injury. Sci Rep 9 : 264, 2019.

16. Jiang L, Wang X, Zhan X, Kang S, Liu H, Luo Y and Lin L: Advance in circular RNA modulation effects of heart failure. Gene X 5: 100036, 2020.

17. Ren S, Lin P, Wang J, Yu H, Lv T, Sun L and Du G: Circula RNAs: Promising molecular biomarkers of human aging-related diseases via functioning as an miRNA sponge. Mol Ther Methods Clin Dev 18: 215-229, 2020.

18. Joshi SS and Coffin CS: Hepatitis B virus lymphotropism: Emerging details and challenges. Biotechnol Genet Eng Rev 34: $139-151,2018$.

19. Szilágyi M, Pös O, Márton É, Buglyó G, Soltész B, Keserú J, Penyige A, Szemes T and Nagy B: Circulating cell-free nucleic acids: Main characteristics and clinical application. Int J Mol Sci 21: 6827, 2020

20. Ho-Xuan H, Glažar P, Latini C, Heizler K, Haase J, Hett R, Anders M, Weichmann F, Bruckmann A, Van den Berg D, et al: Comprehensive analysis of translation from overexpressed circular RNAs reveals pervasive translation from linear transcripts. Nucleic Acids Res 48: 10368-10382, 2020.

21. Que F, Wang H, Luo Y, Cui L, Wei L, Xi Z, Lin Q, Ge Y and Wang W: Comprehensive analysis of differentially expressed circRNAs reveals a colorectal cancer-related ceRNA network. Comput Math Methods Med 2020: 7159340, 2020.

22. Albini A, Magnani E and Noonan DM: The tumor microenvironment: Biology of a complex cellular and tissue society. Q J Nucl Med Mol Imaging 54: 244-248, 2010.

23. Grade M, Ghadimi BM, Varma S, Simon R, Wangsa D, Barenboim-Stapleton L, Liersch T, Becker H, Ried T and Difilippantonio MJ: Aneuploidy-dependent massive deregulation of the cellular transcriptome and apparent divergence of the Wnt/beta-catenin signaling pathway in human rectal carcinomas. Cancer Res 66: 267-282, 2006.

24. Navin NE: The first five years of single-cell cancer genomics and beyond. Genome Res 25: 1499-1507, 2015.

25. Rugbjerg P and Sommer MOA: Overcoming genetic heterogeneity in industrial fermentations. Nat Biotechnol 37: 869-876, 2019.

26. Hashimoto K, Kouno T, Ikawa T, Hayatsu N, Miyajima Y, Yabukami H, Terooatea T, Sasaki T, Suzuki T, Valentine M, et al: Single-cell transcriptomics reveals expansion of cytotoxic CD4 $\mathrm{T}$ cells in supercentenarians. Proc Natl Acad Sci USA 116 24242-24251, 2019

27. Bureau of Medical Administration National Health, Family Planning Comission of the People's Republic of China: Diagnosis, management, and treatment of hepatocellular carcinoma (V2017). Zhonghua Gan Zang Bing Za Zhi 25: 886-895, 2017 (In Chinese).

28. Marioni JC, Mason CE, Mane SM, Stephens M and Gilad Y: RNA-seq: An assessment of technical reproducibility and comparison with gene expression arrays. Genome Res 18: 1509-1517, 2008

29. Jia E, Zhou Y, Liu Z, Wang L, Ouyang T, Pan M, Bai Y and Ge Q: Transcriptomic profiling of circular RNA in different brain regions of Parkinson's disease in a mouse model. Int J Mol Sci 21: 3006,2020

30. Chen Y, Chen Y, Shi C, Huang Z, Zhang Y, Li S, Li Y, Ye J, Yu C Li Z, et al: SOAPnuke: A MapReduce acceleration-supported software for integrated quality control and preprocessing of high-throughput sequencing data. Gigascience 7: 1-6, 2018.

31. Zhang L, Liu C and Dong S: PipeMEM: A framework to speed Up BWA-MEM in spark with low overhead. Genes (Basel) 10: 886, 2019.

32. Giannoulatou E, Park SH, Humphreys DT and Ho JW: Verification and validation of bioinformatics software without a gold standard: A case study of BWA and Bowtie. BMC Bioinformatics 15 (Suppl 16): S15, 2014.
33. Gao Y, Wang J and Zhao F: CIRI: An efficient and unbiased algorithm for de novo circular RNA identification. Genome Biol 16: 4, 2015.

34. Anders S and Huber W: Differential expression analysis for sequence count data. Genome Biol 11: R106, 2010

35. Li S, Chen L, Xu C, Qu X, Qin Z, Gao J, Li J and Liu J: Expression profile and bioinformatics analysis of circular RNAs in acute ischemic stroke in a South Chinese Han population. Sci Rep 10: $10138,2020$.

36. Young MD, Wakefield MJ, Smyth GK and Oshlack A: Gene ontology analysis for RNA-seq: Accounting for selection bias. Genome Biol 11: R14, 2010

37. Mao X, Cai T, Olyarchuk JG and Wei L: Automated genome annotation and pathway identification using the KEGG Orthology $(\mathrm{KO})$ as a controlled vocabulary. Bioinformatics 21 3787-3793, 2005

38. Kanehisa M, Araki M, Goto S, Hattori M, Hirakawa M, Itoh M, Katayama T, Kawashima S, Okuda S, Tokimatsu T and Yamanishi Y: KEGG for linking genomes to life and the environment. Nucleic Acids Res 36: D480-D484, 2008.

39. Shannon P, Markiel A, Ozier O, Baliga NS, Wang JT, Ramage D, Amin N, Schwikowski B and Ideker T: Cytoscape: A software environment for integrated models of biomolecular interaction networks. Genome Res 13: 2498-2504, 2003.

40. Enright AJ, John B, Gaul U, Tuschl T, Sander C and Marks DS MicroRNA targets in Drosophila. Genome Biol 5: R1, 2003.

41. Livak KJ and Schmittgen TD: Analysis of relative gene expression data using real-time quantitative PCR and the 2(-Delta Delta C(T)) method. Methods 25: 402-408, 2001

42. Zhang Z, Fan Y, Xie F, Zhou H, Jin K, Shao L, Shi W, Fang P, Yang B, van Dam H, et al: Breast cancer metastasis suppressor OTUD1 deubiquitinates SMAD7. Nat Commun 8: 2116, 2017.

43. Li X, Yang L and Chen LL: The biogenesis, functions, and challenges of circular RNAs. Mol Cell 71: 428-442, 2018.

44. Thomson DW and Dinger ME: Endogenous microRNA sponges: Evidence and controversy. Nat Rev Genet 17: 272-283, 2016.

45. Kulcheski FR, Christoff AP and Margis R: Circular RNAs are miRNA sponges and can be used as a new class of biomarker. J Biotechnol 238: 42-51, 2016.

46. Li F, Zhang L, Li W, Deng J, Zheng J, An M, Lu J and Zhou Y: Circular RNA ITCH has inhibitory effect on ESCC by suppressing the $\mathrm{Wnt} / \beta$-catenin pathway. Oncotarget 6 : 6001-6013, 2015.

47. Du WW, Yang W, Liu E, Yang Z, Dhaliwal P and Yang BB Foxo3 circular RNA retards cell cycle progression via forming ternary complexes with p21 and CDK2. Nucleic Acids Res 44: 2846-2858, 2016.

48. Tang X, Ren H, Guo M, Qian J, Yang Y and Gu C: Review on circular RNAs and new insights into their roles in cancer. Comput Struct Biotechnol J 19: 910-928, 2021.

49. Xu T, Wu J, Han P, Zhao Z and Song X: Circular RNA expression profiles and features in human tissues: A study using RNA-seq data. BMC Genomics 18 (Suppl 6): 680, 2017.

50. Meng S, Zhou H, Feng Z, Xu Z, Tang Y, Li P and Wu M: CircRNA: Functions and properties of a novel potential biomarker for cancer. Mol Cancer 16: 94, 2017.

51. Shen Y, Bu L, Li R, Chen Z, Tian F, Lu N, Ge Q, Bai Y and Lu Z Screening effective differential expression genes for hepatic carcinoma with metastasis in the peripheral blood mononuclear cells by RNA-seq. Oncotarget 8: 27976-27989, 2017.

52. Li Z, Yang X, Zhang Y, Yang X, Cui X, Zhang Y, Gong W, Bai H, Liu N, Tang Z, et al: A human peripheral blood mononuclear cell (PBMC) engrafted humanized xenograft model for translational immuno-oncology (I-O) research. J Vis Exp 2019.

53. Barbon CM, Janec KJ, Kelner RH, Norton JE and Guinan EC: Alloanergization method for inducing allospecific hyporesponsiveness in PBMC exposed to allostimulation in vitro in the context of costimulatory molecule blockade. Methods Mol Biol 1899: 103-118, 2019.

54. Cheng WC, Saleh F, Abuaisha Karim B, Hughes FJ and Taams LS: Comparative analysis of immune cell subsets in peripheral blood from patients with periodontal disease and healthy controls. Clin Exp Immunol 194: 380-390, 2018.

55. Yuan Y, Zheng S, Li Q, Xiang X, Gao T, Ran P, Sun L, Huang Q, Xie F, Du J and Xiao C: Overexpression of miR-30a in lung adenocarcinoma A549 cell line inhibits migration and invasion via targeting EYA2. Acta Biochim Biophys Sin (Shanghai) 48: $220-228,2016$ 
56. Fu J, Xu X, Kang L, Zhou L, Wang S, Lu J, Cheng L, Fan Z Yuan B, Tian P, et al: miR-30a suppresses breast cancer cell proliferation and migration by targeting Eya2. Biochem Biophys Res Commun 445: 314-319, 2014.

57. Wang T, Chen G, Ma X, Yang Y, Chen Y, Peng Y, Bai Z, Zhang Z, Pei $\mathrm{H}$ and Guo W: MiR-30a regulates cancer cell response to chemotherapy through SNAI1/IRS1/AKT pathway. Cell Death Dis 10: 153, 2019.

58. Yang C, Zhang JJ, Peng YP, Zhu Y, Yin LD, Wei JS, Gao WT, Jiang KR and Miao Y: A Yin-Yang 1/miR-30a regulatory circuit modulates autophagy in pancreatic cancer cells. J Transl Med 15: 211, 2017.

59. Chen J, Yu Y, Li S, Liu Y, Zhou S, Cao S, Yin J and Li G: MicroRNA-30a ameliorates hepatic fibrosis by inhibiting Beclin1-mediated autophagy. J Cell Mol Med 21: 3679-3692, 2017.
60. Ni W, Zhang Y, Zhan Z, Ye F, Liang Y, Huang J, Chen K, Chen L and Ding Y: A novel lncRNA uc.134 represses hepatocellular carcinoma progression by inhibiting CUL4A-mediated ubiquitination of LATS1. J Hematol Oncol 10: 91, 2017.

61. Wang YS, Du L, Liang X, Meng P, Bi L, Wang YL, Wang $C$ and Tang B: Sirtuin 4 depletion promotes hepatocellular carcinoma tumorigenesis through regulating adenosine-monophosphate-activated protein kinase alpha/mammalian target of rapamycin axis in mice. Hepatology 69: 1614-1631, 2019.

c. (i) $९$ This work is licensed under a Creative Commons Attribution-NonCommercial-NoDerivatives 4.0 International (CC BY-NC-ND 4.0) License. 